\title{
Low Grade Malignant Peripheral Nerve Sheath Tumor
}

National Cancer Institute

\section{Source}

National Cancer Institute. Low Grade Malignant Peripheral Nerve Sheath Tumor. NCI

Thesaurus. Code C9026.

A malignant peripheral nerve sheath tumor that is low grade. 